\title{
On Oscillatory Behavior of Solutions of Third-Order Nonlinear Differential Equations
}

\author{
G. Narender ${ }^{1}$, V.V.L. Deepthi ${ }^{2}$, S. Indira ${ }^{3}$ \\ ${ }^{1,2,3}$ CVR College of Engineering, Department of Humanities \& Sciences, \\ Vatunagar, Mangalpalli(V), Ibrahimpatnam(M), R.R.Dist, Telangana State, India
}

*Corresponding Author: G Narender, Asst Prof. Dept. of Mathematics, CVR College of Engineering, Hyderabad, India

\begin{abstract}
In this paper we investigate the behavior of the bounded solutions of a class of third order nonlinear differential equations $\left(r(x)\left(r(x) y^{1}(x)\right)^{1}\right)^{1}+q(x) y^{1}(x)+p(x) y^{\lambda}(x)=0$ has been studied with $p(x)<0, q(x) \geq 0, q^{1}(x) \geq 0$ for $x \in(a, \infty)$.Also the necessary and sufficient condition for any of its solutions to be oscillatory was considered.
\end{abstract}

Keywords: Oscillatory solution, nonoscillatory solution, oscillation, nonlinear differential equations.

\section{INTRODUCTION}

The aim of this paper is study the properties of oscillatory solutions of nonlinear differential equations of third order

$$
\left(r(x)\left(r(x) y^{1}(x)\right)^{1}\right)^{1}+q(x) y^{1}(x)+p(x) y^{\lambda}(x)=0, \lambda>0
$$

Where $p(x), q(x), r(x)$ are continuous functions, with $r(x)>0$ on the interval $I \in(a, \infty),-\infty<a$ and $\lambda$ is positive and is a ratio of two odd integers. The equation (1.1) can be termed as such super linear, linear and sublinear according as $\lambda>1, \lambda=1$ and $\lambda<1$ respectively. A nontrivial solution of (1.1) is said to be oscillatory if it has zeros for arbitrarily large values of the independent variable, otherwise it is said to be nonoscillatory. In the linear case with $\lambda=1$ and $r(x)=1$ the properties of oscillatory solutions are discussed in Hanan [01], Lazer [02] and Swanson [03]. The associated nonlinear equations with $r(x)=1$ is the subject discussed by Waltman [04], Heidal [05], Gragus and Venko [06], N.Parhi and S.Parhi [07] have discussed the equation (1.1) with $q(x)=0$ in the form

$$
y^{111}(x)+p(x) y(x)=0
$$

Also the behavior of solutions of the equations $y^{111}(x)+p(x) y^{11}(x)+q(x) y^{1}(x)+r(x) y^{\lambda}(x)=0(1.3)$ was considered for oscillation, nonoscillation and asymptotic behavior by L.Erbe[08] via a second order equation.

In the discussion of the oscillatory solutions of (1.1)made in the section no recourse has been taken to second order equations as well as no change of variables incorporated. The results obtained generalize many theorems mentioned in the above references. To the best of our knowledge nothing is known regarding the behavior of the bounded solutions of a class of third order nonlinear differential equation.

\section{MAIN RESUltS}

In this section we study the equation

$$
\left(r(x)\left(r(x) y^{1}(x)\right)^{1}\right)^{1}+q(x) y^{1}(x)+p(x) y^{\lambda}(x)=0
$$


with $p(x)<0, q(x) \geq 0, q^{1}(x) \geq 0$ for $x \in(a, \infty)$. We prove a theorem on bounded solutions to be oscillatory and a theorem on necessary and sufficient conditions that a solution of equation (2.1.1) to be oscillatory. We first prove a lemma that is needed for the purpose.

\subsection{LEMMA}

Let

$y(x) \in C^{3}\left(\left[x_{0}, \infty\right)\right)>0$

$r(x) \in C\left(\left[x_{0}, \infty\right)\right), r(x)>0$ and bounded for $x \in\left[x_{0}, \infty\right)$

Then

$y(x)>0,\left(r y^{1}(\mathrm{x})\right)<0$ and $\left(r\left(r y^{1}\right)^{1}\right)^{1}(x)>0$

Cannothold for all $x \geq x_{0}$.

\section{PROOF:-}

Suppose for contradiction that (2.5) holds. Consider $\left(r\left(r y^{1}\right)^{1}\right)^{1}(x)>0$ for $x \geq x_{0}$.

This implies $r(x)\left(r y^{1}\right)^{1}(x)$ increase for all $x \geq x_{0}$.

Since $r(x)$ is positive and bounded we have, $\left(r y^{1}\right)^{1}(x)$ increases for $x \in\left[x_{0}, \infty\right)$.

Suppose

$\left(r y^{1}\right)^{1}(x)>0$,

which implies

$r(x) y^{1}(x)$ increases for $x \in\left[x_{0}, \infty\right)$

Let $\left(r y^{1}\right)^{1}(x)>k$ for some $k>0$.

Integrating between $x_{0}$ and $x$,we get

$r(x) y^{1}(x)>k\left(x-x_{0}\right)+r\left(x_{0}\right) y^{1}\left(x_{0}\right)$

The inequality holds in case $(r y)^{1}(x)>0$, which is a contradiction to our supposition that (2.5) holds.

Now suppose

$\left(r y^{1}\right)^{1}(x)<k$ for some $\mathrm{k}<0, x \in\left[x_{0}, \infty\right)$

Integrating between $x_{0}$ and $x$,we obtain

$r(x) y^{1}(x)<k\left(x-x_{0}\right)+r\left(x_{0}\right) y^{1}\left(x_{0}\right)$

$<0$, for sufficiently large $x$.

Since $r(x)$ is positive and bounded for $x \in\left[x_{0}, \infty\right)$ the inequality (2.11) implies

$y^{1}(x)<0$ for $x \in\left[x_{0}, \infty\right)$ and $y(x)$ decreases.

Suppose

$y(t)>0$ for $x \in\left[x_{0}, \infty\right)$

Let $y^{1}(x)<m$ for some $m<0$ for $x \in\left[x_{0}, \infty\right)$. 
Multiplying with $y(x)$ both sides and integrating between $x_{0}$ and $x$, we obtain

$$
\begin{aligned}
& \int_{x_{0}}^{x} y(s) y^{1}(s) d s<m \int_{x_{0}}^{x} y(s) d s \\
& \frac{y^{2}(x)}{2}<m \int_{x_{0}}^{x} y(s) d s+\frac{y^{2}\left(x_{0}\right)}{2}
\end{aligned}
$$

The left side of (2.13) is positive. While the right side is negative for sufficiently large $x$. So $y(x)>0$ cannot hold for all $x \in\left[x_{0}, \infty\right)$. This is a contradiction to our supposition, that (2.5) holds.

Suppose now that

$$
\left(r\left(r y^{1}\right)^{1}\right)^{1}(x)=0 \text { for } x \geq x_{0}
$$

This implies

$$
r(x)\left(r y^{1}\right)^{1}(x)=k_{1} \text {, a constant. }
$$

Since $r(x)>0$ and bounded for $x>x_{0}(2.14)$ implies

$\left(r y^{1}\right)^{1}(x)=k_{2}$, a constant.

Incase $\left(r y^{1}\right)^{1}(x)=k_{2}>0$, an integration produces

$$
r(x) y^{1}(x)=k_{2}\left(x-x_{0}\right)+r\left(x_{0}\right) y^{1}\left(x_{0}\right)
$$

$>0$, for sufficiently large $x$.

This is a contradiction to our supposition.

Incase $\left(r y^{1}\right)^{1}(x)=k_{2}<0$, an integration produces

$r(x) y^{1}(x)=k_{2}\left(x-x_{0}\right)+r\left(x_{0}\right) y^{1}\left(x_{0}\right)$

$<0$, for sufficiently large $x$.

This implies $y^{1}(x)<0$ since $r(x)>0$ and bounded.

Let $y^{1}(x)<k$, for some $k<0$.

An integration between $x_{0}$ to $x$ produces

$$
\begin{aligned}
& y(x)<k\left(x-x_{0}\right)+y\left(x_{0}\right) \\
& <0
\end{aligned}
$$

Which is a contradiction to our supposition that (2.5) holds. Hence the Lemma.

The main objective of this paper is to prove the following:-

\subsection{THEOREM}

Let $p(x)<0, q(x) \geq 0, q^{1}(x) \geq 0, r(x)>0$ for $x \in(a, \infty)$

$r(x), q(x)$ be bounded on $(a, \infty)$

and

$$
\int_{x_{0}}^{\infty} p(s) d s=-\infty, x_{0}>a
$$


Then every bounded solution of (2.18) on $x \in\left[x_{0}, \infty\right)$ is oscillatory on this interval.

\section{PROOF:-}

Assume without lossof generality that $y(x)>0$ be bounded on $\left[x_{0}, \infty\right), x_{0}>a$.

Three cases arise that depend upon the sign of $r(x) y^{1}(x)$.

\section{CASE(i):}

$$
r(x) y^{1}(x)>0 x \geq X \geq x_{0}
$$

Integrating (2.1) between $x_{0}$ and $x$, we get

$$
r(x)\left(r y^{1}\right)^{1}(x)+q(x) y(x)+\int_{x_{0}}^{x} p(s) y^{\lambda-1}(s)-q^{1}(s) y(s) d s=k
$$

Where

$k=r\left(x_{0}\right)\left(r y^{1}\right)^{1}\left(x_{0}\right)+q\left(x_{0}\right) y\left(x_{0}\right)$, a constant.

Using (2.21), (2.22) and (2.23) and that $y(x)$ bounded,implies $\left(r y^{1}\right)^{1}(x) \rightarrow \infty$ as $t \rightarrow \infty$

Therefore $y(x)$ cannot be bounded on $x \in\left[x_{0}, \infty\right)$, which is a contradiction.

\section{CASE(ii):-}

$$
r(x) y^{1}(x) \leq 0 \text { for } x \geq X \geq x_{0} .
$$

Writing equation (2.1) as

$$
\left(r(x)\left(r(x) y^{1}(x)\right)^{1}\right)^{1}=-q(x) y^{1}(x)-p(x) y^{\lambda}(x)(2.25)>-q(x) r(x) y^{1}(x)-p(x) y^{\lambda}(x)
$$

$>0$ for $x \geq X$, using (2.21).

But by Lemma 2.1 this is impossible.

\section{CASE(iii):-}

$\left(r y^{1}\right)(x)$ has infinitely many null points at which it changes its sign.

Let $y(x)>k>0$, then from (2.24) we obtain,

$\left(r y^{1}\right)^{1}(x)>0$ for $x \geq X \geq x_{0}$

and this implies that $\left(r y^{1}\right)(x)>0$ increases for $x \geq X$.

This is a contradiction with the case $\left(r y^{1}\right)(x)$ is oscillatory.

Therefore $\underset{x \rightarrow \infty}{\operatorname{Liminf}} y(x)=0$.

Since $r(x)$ is positive and bounded.

Suppose,$\underset{x \rightarrow \infty}{\operatorname{Liminf}} y(x)=0$,

We have the following subcases.

\section{Subcase (i):-}

Let

$$
\int_{x_{0}}^{\infty}\left[p(s) y^{\lambda-1}(s)-q^{1}(s)\right] y(s) d s=\infty
$$


Then [2.25] implies that

$\left(r y^{1}\right)^{1}(x)>0$ for $x \geq X \geq x_{0} \mathrm{~T}$.

This is contradiction to the oscillatoricity of $\left(r y^{1}\right)(x)$.

\section{Subcase(ii):-}

Let

$0 \leq-\int_{x_{0}}^{\infty}\left[p(s) y^{\lambda-1}(s)-q^{1}(s)\right] y(s) d s<\infty$

Let $\left\langle x_{i}\right\rangle_{i=1}^{\infty}, x_{i} \rightarrow \infty$ for $i \rightarrow \infty$, be a sequence of points at which $\left(r y^{1}\left(x_{i}\right)\right)=0$ and $\left(r y^{1}\right)^{1}\left(x_{i}\right)>0$.

It follows from (2.22) that $\left(r y^{1}\right)^{1}\left(x_{i}\right)$ is bounded on $\left[x_{0}, \infty\right)$.

Multiplying equation (2.1) by the solution $y(x)$ and integrating from $x_{i}$ to $x$, we obtain

$\int_{x_{i}}^{x}\left(r\left(r y^{1}\right)^{1}\right)^{1}(s) y(s) d s+\int_{x_{i}}^{x} q(s) y^{1}(s) y(s) d s+\int_{x_{i}}^{x} p(s) y^{\lambda+1}(s) d s=0$

Integrating by parts yields

$$
\begin{aligned}
& r(x) y(x)\left(r y^{1}\right)^{1}(x)-\frac{1}{2}\left(r y^{1}\right)^{2}(x)+\frac{1}{2} q(x)\left(y^{1}\right)^{2}(x)+\int_{x_{i}}^{x}\left[p(s) y^{\lambda-1}(s)-\frac{1}{2} q^{1}(s)\right] y^{2}(s) d s \\
& =r\left(x_{i}\right) y\left(x_{i}\right)\left(r y^{1}\right)^{1}\left(x_{i}\right)-\frac{1}{2}\left(r y^{1}\right)^{2}\left(x_{i}\right)+\frac{1}{2} q\left(x_{i}\right) y^{2}\left(x_{i}\right), i=1,2,3, \ldots \ldots \ldots \ldots
\end{aligned}
$$

We write the above equation in the form

$$
r(x) y(x)\left(r y^{1}\right)^{1}(x)-\frac{1}{2}\left(r y^{1}\right)^{2}(x)+\frac{1}{2} q(x)\left(y^{1}\right)^{2}(x)+\int_{x_{i}}^{x}\left[p(s) y^{\lambda-1}(s)-\frac{1}{2} q^{1}(s)\right] y^{2}(s) d s=k_{i}
$$

Where $k_{i}=r\left(x_{i}\right) y\left(x_{i}\right)\left(r y^{1}\right)^{1}\left(x_{i}\right)-\frac{1}{2}\left(r y^{1}\right)^{2}\left(x_{i}\right)+\frac{1}{2} q\left(x_{i}\right) y^{2}\left(x_{i}\right), i=1,2,3, \ldots \ldots \ldots \ldots$

From this we obtain for $\left(r y^{1}\right)^{1}\left(x_{i}\right), i=1,2,3, \ldots \ldots \ldots$

$\left(r y^{1}\right)^{1}\left(x_{i}\right)=\frac{k_{i}}{r\left(x_{i}\right) y\left(x_{i}\right)}-\frac{1}{2} \frac{q\left(x_{i}\right)\left(y^{1}\left(x_{i}\right)\right)^{2}}{r\left(x_{i}\right) y\left(x_{i}\right)}-\frac{1}{r\left(x_{i}\right) y\left(x_{i}\right)} \int_{x_{i}}^{x}\left[p(s) y^{\lambda-1}(s)-\frac{1}{2} q^{1}(s)\right] y^{2}(s) d s$.

From this it follows that $x_{i} \rightarrow \infty,\left(r y^{1}\right)^{1}\left(x_{i}\right) \rightarrow \infty$.

Which is a contradiction with the boundedness of $\left\{\left(r y^{1}\right)^{1}\left(x_{i}\right)\right\}$. this complete the proof.

\subsection{LEMMA}

Let

$p(x)<0, q(x) \geq 0, q^{1}(x) \geq 0, r(x)>0$

for $x \in(a, \infty)$

$r(x), q(x)$ be on bounded $x \in(a, \infty)$ 
and

$\int_{x_{0}}^{\infty} p(s) d s=-\infty, x_{0}>a$

Then for every $y(x)$ of (2.1) with the property $y(x)>0$ for $x>x_{0}$, there exists $X \geq x_{0}$ such that for $x \geq X$ the inequality

$r(x) y(x)\left(r y^{1}\right)^{1}(x)-\frac{1}{2}\left(y^{1}(x)\right)^{2}+q(x) y^{2}(x)>0$ holds.

PROOF:-

Let $y(x)>0$ for $x \geq x_{0}$. Then there are three cases for $\left(r y^{1}\right)(x)$.

CASE(i):-

$\left(r y^{1}\right)(x)>0$ for $x \geq x_{0}$.

It follows from (2.32) with $x_{i}=x_{0}$ that there exists $X \geq x_{0}$ such that for all $x \geq X$, the inequality (2.37) holds.

\section{CASE(ii):-}

$\left(r y^{1}\right)(x) \leq 0$ for $x \geq x_{0}$

Writing (2.1) as

$$
\begin{aligned}
\left(r(x)\left(r(x) y^{1}(x)\right)^{1}\right)^{1}= & -q(x) y^{1}(x)-p(x) y^{\lambda}(x) \\
& >0 \text { using }(2.34)
\end{aligned}
$$

But using Lemma 2.2 this is not possible.

\section{CASE(iii):-}

$\left(r y^{1}\right)(x)$ has on $\left[x_{0}, \infty\right)$ at least two null points at which it changes sign.

At one of the null points we have $\left(r y^{1}\right)^{1}(x) \geq 0$.

Let $x=X_{i}$. It follows from (2.32) with $x_{i}=X$ that

$$
k_{i}=r\left(X_{i}\right) y\left(X_{i}\right)\left(r y^{1}\right)^{1}\left(X_{i}\right)+\frac{1}{2} q\left(X_{i}\right) y^{2}\left(X_{i}\right), i=1,2,3, \ldots \ldots \geq 0
$$

Such that (2.37) holds for $x>X$. Thus the lemma is proved.

\subsection{THEOREM}

Let

$$
p(x)<0, q(x) \geq 0, q^{1}(x) \geq 0, r(x)>0
$$

for $x \in(a, \infty)$

$r(x), q(x)$ be on bounded $x \in(a, \infty)$

And $\int_{x_{0}}^{\infty} p(s) d s=-\infty, x_{0}>a$

Then a necessary and sufficient condition for the solution $y(x)$ of $(2.1)$ define on $\left[x_{0}, \infty\right), x_{0}>a$ to be oscillatory on $\left[x_{0}, \infty\right)$ is that 
$r(x) y(x)\left(r y^{1}\right)^{1}(x)-\frac{1}{2}\left(r y^{1}\right)^{2}(x)+\frac{1}{2} q(x) y^{2}(x)<0$ for $x \geq X \geq x_{0}$

\section{PROOF:-}

We prove the condition (2.43) is sufficient. Let $y(x)$ be a solution of (2.1) which satisfies the condition (2.43). Let us assume without loss of generality that $y(x)>0$ for $x \geq X$. But then by lemma 2.33 there exists $X_{1} \geq x_{0}$ such that (2.42.) holds for $x \geq X_{1}$ and this contradicts (2.43). Thus must be oscillatory.

We shall now prove the condition (2.43) is necessary. That is we must prove that an oscillatory solution in $\left[x_{0}, \infty\right)$ fulfils the condition (2.43). Let $y(x)$ be an oscillatory solution of $(2.1)$ on $\left[x_{0}, \infty\right)$ and let $x_{i}, i=1,2,3, \ldots \ldots \ldots \ldots \ldots . . . .$. be its null points on $\left[x_{0}, \infty\right)$. Let us denote $G(y(x))$ by

$G(y(x)):=r(x) y(x)\left(r y^{1}\right)^{1}(x)-\frac{1}{2}\left(r y^{1}\right)^{2}(x)+\frac{1}{2} q(x) y^{2}(x)$

Equation (2.30) can be written as

$$
\begin{aligned}
& G(y(x)):= \\
& G\left(y\left(x_{i}\right)\right)-\int_{x_{i}}^{x}\left[p(s) y^{\lambda-1}(s)-\frac{1}{2} q^{1}(s)\right] y^{2}(s) d s \\
& \frac{d G(y(x))}{d x}=-\left[p(x) y^{\lambda-1}(x)-\frac{1}{2} q^{1}(x)\right] y^{2}(x) \\
& >0 \text { on }[x, \infty) \text { using }(2.1)
\end{aligned}
$$

(2.44) implies that $G(y(x))$ is increasing on $\left[x_{1}, \infty\right)$ and thus

$$
G(y(x)):=r\left(x_{i}\right) y\left(x_{i}\right)\left(r y^{1}\right)^{1}\left(x_{i}\right)-\frac{1}{2}\left(r y^{1}\right)^{2}\left(x_{i}\right)+\frac{1}{2} q(x) y^{2}\left(x_{i}\right)<0 \text { for } i=1,2,3, \ldots \ldots \ldots . . .
$$

and so (2.42) holds. Hence the proof.

\section{CONCLUSiON}

In this paper, we considered the third order non-linear homogeneous differential equations. Let $y(x)$ be a solution of (2.1) with the property $y\left(x_{0}\right)=0,\left(r y^{1}\right)\left(x_{0}\right)=0,\left(r y^{1}\right)^{1}\left(x_{0}\right)>0$ and let the hypothesis of theorem (2.37)be satisfied. Then $y(x)>0$ for $x>x_{0}$.

\section{ACKNOWLEDGMENT}

My Sincere thanks to my supervisor Professor G. Ramakrishna Rao for his guidance and continuous support to complete this work.

\section{REFERENCES}

[1] M.Hanan, "Oscillation criteria for third order linear differential equations," Pacific J.Math; vol.11, pp. 919944,1961.

[2] A. C. Lazer, "The behaviour of solutions of the differential equation $y^{111}+p y^{1}+q y=0$," Pacific Journal Math., vol.17, pp.435 -466, 1966

[3] C.A. Swanson, Comparision and oscillation theory of linear differential equation," Academipress, Newyork, 1968.

[4] Paul Waltman, "Oscillation criteria for third order nonlinear differential equations," Pacific Journal of Mathematics, vol.18, pp.384-389,1966.

[5] J. W. Heidel. and Don B. Hinton, "The existence of oscillatory solutions for a Nonlinear differential equation,” SIAM J.Math. Anal. Vol3, No.2,pp.344-351,1972. 
[6] M.Gregus and J.Vencko, on oscillatory properties of solutions of certain nonlinear third order differential equation,” czechoslovak Mathematical Journal, vol.42 (117), pp.675-684, 1992.

[7] N.Parhi and S.Parhi, "Oscillation and non oscillation theorems for non homogenous third order differential equations,” Bull. Inst. of Math. Academia Sinica, vol.11, pp.125-139,1983.

[8] Erbe, "Oscillation, Nonoscillation and Asymptotic behaviour of third order Nonlinear Differential equations,” Anali di Mate puraed Appl. Vol. CX,pp.373-393, (1976)

[9] J. Anders, "Boundedness of solutions ofthe third order differential equation with oscillatory restoring and forcing terms," Czechoslovak Mathematical Journal, vol.36 (III), pp.1-6, 1986.

[10] J.H.Barrett, "Oscillation theory of ordinary linear differential equation," Advances in Maths. , vol.3, pp. 415-509, 1969.

[11] N. Pahri and P. Das, "Asymptotic property of solutions of a class of third-order differential equations," proc. Amer. Math. Soc. Vol.110,pp.387-393, 1990

[12] V.S.H.Rao and R.S.Dahiya, " properties of solutions a class of third order linear differential equations," periodica Mathematica Hungarica20, (1989)', 177-184

[13] L.H.Erbe and V.S.H.Rao, "Nonoscillation Results for third order Nonlinear Differential equations, JMAA, vol.125, pp.471-482, (1987)

[14] L. Erbe and V. Sreehari Rao, "Nonoscillationcritria for forced second order Nonlinear Equations," Journal of Mathematical Analysis and, Applications, Vol. 97,pp.202-213, Nov.1983

[15] L. Erbe and V.Sreehari Rao, "On the generalizedEmdn- Fowler and Thomas-Ferni Differential equations," Bull. Alld. Math. SeoVol. 5, pp. 21-78, 1990.

[16] L.Erbe, V.Sreehari Rao and K.V.V. Sehsagiri Rao, "Non- oscillation and asymptotic properties for a class of forced second order nonlinear equations, Math. Proc.camb. Phil.Soc. vol.95, pp.155-163, 1984.

[17] HUGO TEUFEL, Jr. Forced Second Order Nonlinear Oscillation, Jour. Math Anal and Appl. Vol 40, No. 1(1972) $140-152$.

[18] KAZIMIERZ ZIMA (RZESZO'W): A certain theorem on the oscillation of solutions of equation. $\left(p(t) x^{1}\right)^{1}+q(t) x=0$; Annalessecetatis Mathematica Polonae, Series 1: XXI (1979, $391-393$.

[19] PAUL WALTMAN, Oscillation riteria for third order nonlinear differential equations, Pacific Journal of Mathematics 18 (1966) $384-389$.

[20] S.D.TELLIAFERRO, Asymptotic behavior of solutions of $y^{11}=q(t) y$.J.Math.Anal.Appl. 66 (1978) 95 $-134$.

[21] C.A.SWANSON, Comparision and oscillation theory of linear differential equation, Academic press, Newyork, 1968.

[22] V.S.H.RAO and R.S.DAHIYA, properties of solutions of a class of third order linear differential equations, Periodica Mathematica Hungarica 20, (1989), 177 - 184.

[23] N.PARHI, On oscillatory solutions of a forced second order nonlinear differential equations. EquazioniDifferenzaliordinarie, Vol. XVI (1979) 357 - 360.

[24] N.PARHI and S.K.NAYAK, on nonoscillatory behavior of solutions of nonlinear differential equations, Atti Della Acad. National Del Lincei, 65 (1978), 58 - 62. 


\section{AUTHORS' BIOGRAPHY}
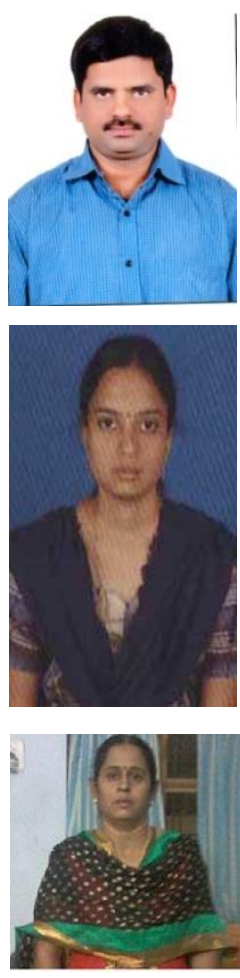

Mr. G. Narender, He is working as a Assistant Professor in Mathematics, CVR College of Engineering, Mangallpalli(V), Ibrahimpatnam(M), Rangaraddy Dist. His field of interest in research and teaching in applied mathematics

V.V.L.Deepthi, She is working as a Assistant Professor in Mathematics, CVR College of Engineering, Mangallpalli(V), Ibrahimpatnam(M), Rangaraddy Dist.

S.Indira, She is working as a Assistant Professor in Mathematics, CVR College of Engineering, Mangallpalli(V), Ibrahimpatnam(M), Rangaraddy Dist.

Citation: G. Narender et al., " On Oscillatory Behavior of Solutions of Third-Order Nonlinear Differential Equations ", International Journal of Scientific and Innovative Mathematical Research, vol. 5, no. 6, p. 3442, 2017., http://dx.doi.org/10.20431/2347-3142.0506004

Copyright: (ㅇ 2017 Authors. This is an open-access article distributed under the terms of the Creative Commons Attribution License, which permits unrestricted use, distribution, and reproduction in any medium, provided the original author and source are credited. 